Published in final edited form as:

Infect Dis Clin North Am. 2018 March ; 32(1): 145-162. doi:10.1016/j.idc.2017.10.007.

\title{
Encephalitis in US Children
}

\author{
Kevin Messacar, MD, \\ Assistant Professor, Dept of Pediatrics, University of Colorado/Children's Hospital Colorado, \\ Aurora, CO, USA \\ Marc Fischer, MD, MPH, \\ Arboviral Diseases Branch, Centers for Disease Control and Prevention, Fort Collins, CO, USA \\ Samuel R Dominguez, MD, PhD, \\ Associate Professor, Dept of Pediatrics, University of Colorado/Children's Hospital Colorado, \\ Aurora, CO, USA \\ Kenneth L Tyler, MD, and \\ Louise Baum Endowed Chair of Neurology and Chairman Dept. of Neurology, Professor of \\ Medicine and Immunology-Microbiology, University of Colorado, Aurora, CO USA \\ Mark J Abzug, MD \\ Professor, Dept of Pediatrics, University of Colorado/Children's Hospital Colorado, Aurora, CO, \\ USA
}

\section{SYNOPSIS}

Encephalitis is an uncommon but severe disease characterized by neurologic dysfunction with central nervous system inflammation. Children with encephalitis should receive supportive care and empiric therapies for common and treatable causes while prioritizing diagnostic evaluation for common, treatable, and high-risk conditions. Even with an extensive diagnostic work-up, an infectious etiology is identified in less than half of cases, suggesting a role for post-infectious or non-infectious processes.

\section{Keywords}

Encephalitis; Meningoencephalitis; Myelitis; Herpes Simplex Virus; Enterovirus; Mycoplasma; anti-NMDA; Arbovirus

CORRESPONDING AUTHOR: KM: kevin.messacar@childrenscolorado.org 13123 E 16 ${ }^{\text {th }}$ Ave, B055, Aurora, CO 80045.

KM: kevin.messacar@ childrencolorado.org. B055, 13123 E $16^{\text {th }}$ Ave, B055, Aurora, CO 80045. 720-777- 6627

MF: mfischer@cdc.gov.3156 Rampart Road, Fort Collins, CO 80521. 970-221-6489

SRD: Samuel.dominguez@childrenscolorado.org. B055, 13123 E 16 ${ }^{\text {th }}$ Ave, B055, Aurora, CO 80045. 720- 777-8883

KLT: ken.tyler@ucdenver.edu.12700 E19 $19^{\text {th }}$ Ave, B182, Aurora, CO 80045

MJA: mark.abzug@childrenscolorado.org. B055, 13123 E $16^{\text {th }}$ Ave, B055, Aurora, CO 80045. 720-777-6389

DISCLOSURE STATEMENT

None of the authors have any conflicts of interest to disclose. KM receives salary support from NIH 1K23AI128069-01. KLT receives grant support from NIH 5R33AI101064, R56NS101208, R21NS103186, VA MERIT 5I01BX000963. The views expressed in this document are those of the authors and do not necessarily reflect those of the Centers for Disease Control and Prevention.

Publisher's Disclaimer: This is a PDF file of an unedited manuscript that has been accepted for publication. As a service to our customers we are providing this early version of the manuscript. The manuscript will undergo copyediting, typesetting, and review of the resulting proof before it is published in its final citable form. Please note that during the production process errors may be discovered which could affect the content, and all legal disclaimers that apply to the journal pertain. 


\section{Introduction}

Encephalitis is a rare, but serious, condition of neurologic dysfunction due to inflammation of the brain parenchyma. A wide variety of infectious and non-infectious etiologies are associated with encephalitis, though the cause in more than half of cases remains unexplained despite extensive testing. Given the heterogeneous and wide differential diagnosis, epidemiological, clinical, laboratory, and radiographical factors are necessary to guide the diagnostic evaluation and treatment. This review focuses on the most common etiologies of acute encephalitis in previously healthy children in the US and introduces a practical approach to prioritizing diagnostic evaluation and treatment.

\section{Case Definition}

Brain parenchymal inflammation associated with neurologic dysfunction is the strict definition of confirmed encephalitis ${ }^{1}$. However, due to the rarity of pre-mortem brain biopsy specimens available for histopathologic confirmation (particularly in children), clinical correlates are used to infer evidence of probable brain inflammation. Wide variability in criteria utilized and emphasized by pediatric neurologists and infectious diseases subspecialists was previously used to infer a clinical diagnosis of encephalitis ${ }^{2}$. In 2013, the International Encephalitis Consortium (IEC) created simplified consensus diagnostic criteria for a standardized case definition of encephalitis and encephalopathy of presumed infectious or autoimmune etiology ${ }^{3}$. Altered mental status for over 24 hours without an alternative cause is required as evidence of neurologic dysfunction. In addition, supplemental minor criteria must be present ( 2 for possible, $\geq 3$ for probable or confirmed): fever $238^{\circ} \mathrm{C}$ within 72 hours, seizures, new focal neurologic findings, cerebrospinal fluid (CSF) pleocytosis ( 25 white blood cells $/ \mu \mathrm{L}$ ), neuroimaging with brain parenchymal changes or electroencephalogram (EEG) consistent with encephalitis (Figure 1). Confirmed cases require pathologic confirmation on brain biopsy, evidence of infection with a microorganism associated with encephalitis, or laboratory evidence of an autoimmune condition associated with encephalitis.

The IEC case definition combines previously distinct categories of encephalopathy and encephalitis without differentiating infectious from post- or non-infectious processes, which may have important therapeutic implications. In cases with altered mental status $\geq 24$ hours without signs of an inflammatory response (fever, CSF pleocytosis, parenchymal changes on neuroimaging), a clinical diagnosis of encephalopathy, rather than encephalitis, is appropriate. In cases meeting encephalitis criteria with CSF pleocytosis, meningeal signs, or leptomeningeal enhancement, a clinical diagnosis of meningoencephalitis may be more descriptive ${ }^{4}$.

Several factors should be considered when applying the IEC case definition to pediatric patients. Simple and complex febrile seizures are a common occurrence in young children and, in isolation, do not necessitate pursuing a work-up for encephalitis if the child has returned to baseline mental status. Normal CSF white blood cell counts (WBC) in infants are higher than those cited for adults and a $95^{\text {th }}$ percentile cutoff of $\leq 19 \mathrm{WBCs} / \mu \mathrm{L}$ for infants $\leq$ 
month and $₫ \mathrm{WBCs} / \mu \mathrm{L}$ for infants 1-2 months are more commonly used to define pleocytosis in these age groups ${ }^{5}$. Young infants are more likely to have infectious encephalitis without pleocytosis, particularly with enterovirus $(\mathrm{EV} ; \sim 50 \%)$ or human parechovirus (HPeV; pleocytosis uncommon) ${ }^{6-8}$. Therefore, as the IEC criteria suggest, CSF pleocytosis is a supportive, but not necessary criterion for encephalitis, particularly in young infants.

\section{Epidemiology}

Overall, there were 7.3 encephalitis cases per 100,000 person years in the US during 2000$2010^{9}$ with peak incidence in infants $<1$ year $(13.5$ per 100,000$)$ and lowest in children $10-$ 14 years $(4.1$ per 100,000) 9 . 7298 hospital admissions for encephalitis amongst the 44 free standing children's hospitals in the Pediatric Health Information System network in the US occurred from 2004-2013 (mean 18 per hospital annually) ${ }^{10}$.

The reported incidence of encephalitis in children in the US and England increased over the past 10 years $^{9,10}$, which may be partially attributed to increasing use of immunosuppressive therapies and bone marrow and solid organ transplantation associated with an increased risk of encephalitis, as well as improved sensitivity of brain parenchymal imaging using magnetic resonance imaging (MRI). Prior to this era, the number of encephalitis cases was decreasing following introduction of vaccines against poliovirus, measles virus, mumps virus, varicella virus, and pertussis ${ }^{11-13}$. Seasonal increases in the incidence of encephalitis in children occur over the summer to fall months, largely driven by epidemic and endemic circulation of arboviruses and $\mathrm{EVs}^{9}$.

\section{Pathogenesis}

Though poorly understood for some etiologies, a variety of mechanisms contribute to encephalitis. The two main forms of encephalitis are primary infectious encephalitis, resulting from direct invasion of the central nervous system (CNS; most commonly gray matter) by the pathogen, and immune mediated encephalitis, resulting from CNS damage from the immune system (most commonly white matter) ${ }^{14}$. Viruses can invade the CNS via viremia subsequently crossing the blood-brain barrier (eg. arboviruses) or retrograde axonal transport (eg. rabies virus) and infect neurons leading to cytotoxicity (eg. herpes simplex virus; HSV). Additionally, pathogens can cause inflammation leading to tissue damage (eg. West Nile virus; WNV) or cause vasculitis leading to tissue ischemia (eg. varicella zoster virus; VZV), or a combination of these mechanisms ${ }^{15}$. Alternatively, primarily nonneuroinvasive pathogens infecting non-CNS sites (eg. Mycoplasma pneumoniae, influenza virus respiratory infections), neuroinvasive pathogens infecting the CNS (eg. HSV), tumors (eg. ovarian teratomas), and potentially some vaccinations may trigger CNS autoimmunity due to aberrant immune response against brain antigens. Direct CNS viral infection and triggering of immune mediated disease may co-exist, as illustrated with HSV encephalitis cases with subsequent or concurrent anti-N-methyl-D-aspartate receptor (anti-NMDAR) antibodies identified $^{16,17}$. 


\section{Diagnosis}

\section{Overview of Etiologies}

In the clinical setting, an etiology is identified in roughly $50 \%$ of cases of encephalitis in children ${ }^{9,11}$. Of unexplained cases, more than $60 \%$ lack an identifiable cause even with comprehensive research testing using advanced molecular diagnostic technologies ${ }^{18}$. A broad array of infectious, immune-mediated, rheumatologic, endocrinological, neoplastic, and toxicologic causes all may cause or mimic encephalitis. Infectious causes, including viruses, atypical bacteria, fungi, and parasites are most common, with viruses accounting for a majority of infectious encephalitis cases in children (Table 1). Immune mediated causes of encephalitis include demyelinating conditions such as acute disseminated encephalomyelitis (ADEM) and neuronal autoantibody mediated conditions such as anti-NMDAR encephalitis and account for an increasing proportion of unexplained encephalitis cases (Table 2) ${ }^{19}$.

\section{Confirming the Syndromic Diagnosis}

The first step in the diagnostic evaluation of a child with suspected encephalitis is to confirm the syndromic diagnosis by looking for evidence of neurologic dysfunction and brain parenchymal inflammation, while ruling out clinical mimickers (eg. toxic ingestion). A comprehensive, detailed history and physical examination is of utmost importance to characterize neurologic deficits, assess for meningeal signs and symptoms, and elicit risk factors. Typical alterations in speech, behavior, and cognition in older individuals with encephalopathy may be more challenging to detect in infants and young children, who present more commonly with irritability, lethargy, or loss of interest in feeding.

Diagnostic testing should include brain MRI, lumbar puncture, and EEG in all suspected cases of encephalitis (Table 3). MRI of the brain with and without contrast utilizing diffusion weighted, T2-weighted, and FLAIR sequences is the modality of choice to assess for changes consistent with brain parenchymal inflammation. After assessing for contraindications, a lumbar puncture with opening pressure measurement should be performed to obtain CSF for characterization of cell counts with differential, glucose, protein, and diagnostic testing. CSF in viral encephalitis typically has a mild to moderate mononuclear pleocytosis (predominantly lymphocytic, though can be neutrophilic early in course), increased protein, and normal glucose; though early in the course or with EV and $\mathrm{HPeV}$ infections in young infants abnormalities may be absent ${ }^{6-8}$. Repeat lumbar puncture should be considered if persistent or worsening symptoms for repeat diagnostic testing to assess for evolution of these findings. EEG should be used to look for evidence of encephalopathy, localizing signs, characteristic patterns (such as periodic localizing epileptiform discharges; PLEDs) or subclinical seizure activity.

\section{Diagnostic Approach to Identifying an Etiology}

The identification of a specific etiology may allow initiation of effective, targeted therapies for certain treatable etiologies, and limit unnecessary diagnostic testing or empiric therapies. Varying levels of evidence establishing a potential etiology as a cause of encephalitis may be present ${ }^{20}$. For established causes of encephalitis, evidence of an etiologic agent in brain tissue specimens or CSF (eg. HSV DNA), or intrathecal antibody production for pathogens 
where PCR is not the study of choice (eg. West Nile virus IgM antibodies), is considered a confirmed cause. Serologic evidence (eg. serum Bartonella henselae IgM antibodies) without PCR confirmation of an established cause that can sometimes be detected directly in CSF or detection of a not well-established cause in brain tissue or CSF by PCR (eg. HHV-6 DNA) are examples of situations that meet the definition of probable cause. Finding suggestive serologic evidence (eg. West Nile virus IgM antibodies in serum) or detection of a well-established pathogen at a site outside the central nervous system (eg. influenza RNA in respiratory specimens) is considered a possible cause. It is imperative that the clinical presentation and epidemiologic profile are consistent with the etiology detected for all levels of causality to avoid erroneous attribution of causality.

Given the broad array of etiologies of encephalitis, it is essential to prioritize the differential diagnosis for a targeted, staged diagnostic evaluation (Table 4). We advocate a two-tiered approach: i) a. testing for common and/or treatable causes and b. selective testing for etiologies more likely based on risk factors, and ii) broader and/or more invasive testing for unexplained persistent or severe disease. Additionally, testing and reporting to state public health departments should be considered for certain etiologies that carry public health significance (eg. arboviruses, rabies virus, free-living ameba).

\section{Tier 1a: Testing for Common and Treatable Etiologies}

The most common infectious pathogens in children include HSV-1 and 2 and EVs, which can be identified in CSF, blood, and nonsterile sites (skin lesions and either pharynx/rectum for EVs or eye/pharynx/rectum for HSV in neonates). Given that HSV is treatable and carries high morbidity and mortality, all children with encephalitis should be tested for HSV by PCR testing of CSF, with consideration of repeat testing if clinical findings are suggestive and no alternative etiology identified ${ }^{21}$. Testing of non-sterile sites increases sensitivity of detection, particularly for HSV in neonates and for EVs uncommonly found in CSF (eg. EVA71, EV-D68, poliovirus), but must be interpreted with caution ${ }^{22,23}$. Multiplex PCR testing allows for rapid detection of multiple infectious agents, including HSV and EV, using a single test from a small amount of $\mathrm{CSF}^{24}$. Given the similar clinical presentations of infectious causes of encephalitis, rapid syndromic testing for a panel of common pathogens using a small volume of CSF may be advantageous. Bacterial meningitis mimicking meningoencephalitis should be considered in any child presenting with fever, headache, meningeal signs and/or encephalopathy, with blood and CSF sent for bacterial cultures.

\section{Tier 1b: Selected Testing for Etiologies More Likely Based on Risk Factors}

Targeted diagnostic testing can be directed by assessing host factors, epidemiologic factors, and clinical characteristics (Table 1). The patient's age, immune status and vaccinations; seasonality, travel, and exposures; as well as clinical signs and symptoms, and localizing findings on imaging and EEG can all be used to prioritize testing for etiologies for which the patient is particularly at-risk.

Host Factors-In addition to HSV and EV, evaluating for HPeVs in young infants with encephalitis by PCR testing of CSF and potentially blood, throat, and/or rectal samples should be considered ${ }^{8,22}$. Consideration of congenital infections such as cytomegalovirus, 
syphilis, Zika virus, and toxoplasma in neonates based on clinical characteristics also is warranted. Human immunodeficiency virus (HIV) testing by serology should be considered in all patients at risk, with additional RNA PCR testing conducted in adolescents with concern for acute retroviral syndrome or infants in whom serologic testing may be confounded by maternal antibody. Syphilis also should be considered in adolescent patients with serologic screening followed by CSF VDRL testing to confirm neurosyphilis. Mumps, measles, varicella, influenza, and polio viruses, are more likely in children who lack immunization and have travel/exposure risk factors for these pathogens. The diagnostic evaluation of a child with a known or concerning history for immunodeficiency or who is receiving immunosuppressive medications warrants a broader diagnostic work-up for opportunistic pathogens.

Epidemiologic Factors-Seasonality in the spring to fall months, geographic location, and exposure to mosquitoes or ticks can be used to guide testing for arboviruses (eg. $\mathrm{La}$ Crosse virus, West Nile virus, Eastern equine encephalitis virus, Powassan virus, and St Louis encephalitis virus) ${ }^{25}$, rickettsia, borrelia (Lyme Disease), ehrlichia, or anaplasma. In immunocompetent children, these insect-borne pathogens are best assessed by serologic testing of blood and CSF to detect intrathecal antibody production confirming neuroinvasive disease. A travel history to endemic areas warrants additional testing for exotic arboviruses (eg. chikungunya, dengue, Japanese encephalitis, tickborne encephalitis, or Zika viruses). During the winter months, influenza virus, adenovirus, and other seasonal respiratory viruses associated with encephalitis should be evaluated by PCR testing of respiratory specimens. When tuberculosis risk factors are present, tuberculin skin testing or interferongamma release assay testing, a chest radiograph, and CSF mycobacterial culture and PCR should be obtained

Exposures-History of animal contact can help guide testing for rabies virus following bites (esp. bats, skunks), Baylisascaris procyonis following exposure to raccoon feces or latrines, lymphocytic choriomeningitis virus (LCMV) following rodent exposure, and Bartonella henselae following feline exposure. Dietary history can help guide testing for pathogens transmitted via unpasteurized dairy, undercooked meat, seafood, or unwashed vegetables (see Table 4). Recreational activities in freshwater are a risk factor for leptospirosis, and Naeglaeria fowlerii amebic encephalitis (also transmitted via non-sterile sinus rinses $)^{26}$. In contrast, freshwater exposure is typically absent in cases of Balamuthia mandrillaris amebic encephalitis, which can be transmitted by soil exposure ${ }^{27}$. Wet mount of CSF looking for free-living ameba can be conducted only on fresh CSF specimens. Specific PCR testing for ameba in CSF or brain tissue (more sensitive) can be conducted through the Centers for Disease Control and Prevention ${ }^{28}$.

Findings on Physical Examination-The presence of a vesicular rash should prompt HSV, EV, and VZV testing by PCR or viral culture of an unroofed vesicle swab with CSF PCR testing to confirm a diagnosis. Regional lymphadenopathy can be suggestive of Bartonella henselae, whereas diffuse adenopathy may warrant serologic evaluation for systemic viral illnesses such as HIV, EBV, or CMV. Ophthalmologic exam can detect characteristic retinitis or keratitis patterns which may prompt testing for WNV, 
cytomegalovirus, or Bartonella henselae. Respiratory symptoms should prompt testing for respiratory pathogens associated with encephalitis, including influenza virus, Mycoplasma pneumoniae, and adenovirus, many of which can be assessed through PCR testing of respiratory specimens. Parotitis is most commonly found with mumps virus, but can be seen with HIV, CMV, EBV, influenza virus and other respiratory viruses also associated with encephalitis. Hydrophobia and hypersalivation are suggestive, though not sensitive, signs of rabies encephalitis. Prominent behavior or psychiatric changes, abnormal limb movements, and dysautonomia should prompt testing for neuronal autoantibodies, including antiNMDAR, in serum and CSF.

Findings on Diagnostic Studies-Certain laboratory, electrophysiological, and imaging patterns can be suggestive of particular etiologies. Eosinophilia in the CSF is always an abnormal finding and should prompt testing for parasites (eg. Angiostrogylus cantonensis, Taenia solium, Baylisascaris procyonis, Toxocara canis/catis, Toxoplasma gondii, free-living ameba), tuberculosis or fungal etiologies, if the patient has exposure risk factors. EEG with PLEDs or temporal lobe-localizing EEG activity or neuroimaging are suggestive, but not specific for HSV, and can be seen with a spectrum of infectious pathogens, including tuberculosis and $\mathrm{VZV}^{29}$. Ring-enhancing lesions can be associated with Toxoplasma gondii, ameba, fungi, and tuberculosis. Respiratory viruses, especially influenza virus, have been associated with thalamic and basal ganglia lesions ${ }^{30}$. Rhomboencephalitis, or brainstem involvement, has been described with Listeria monocytogenes and mycobacteria, as well as EVs, particularly EV-A71, and HSV. Cerebellitis can follow a variety of viral infections, but is most commonly seen with VZV in areas without widespread VZV vaccination. The presence of myelitis with encephalitis on imaging is suggestive of an enterovirus (eg. EV-A71 or EV-D68) or flavivirus (eg. WNV, Japanese encephalitis virus $)^{31,32}$. A presumptive diagnosis of ADEM can be made based on neuroimaging findings of multifocal, diffuse, poorly demarcated demyelinating lesions in the white matter or deep grey matter in the setting of encephalitis ${ }^{33}$.

\section{Tier 2: Broader, More Invasive Testing}

When no cause has been identified despite clinical testing for common, treatable, and at-risk etiologies, and the patient is not improving or has severe disease, consideration should be given to broader and more invasive diagnostic testing. This is particularly important in immunocompromised children, as the identification of an etiology is challenging given the broad differential diagnosis. In addition to further pathogen-directed testing, metagenomic sequencing of CSF or brain tissue enables unbiased assessment for bacteria, viruses, fungi, and parasites not suspected or detected using traditional clinical testing, as well as novel pathogens. Potentially treatable pathogens, such as Leptospira, Brucella, and Balamuthia mandrillaris, as well as untreatable pathogens, such as astroviruses and novel viruses, that were not suspected clinically have been identified using this technology ${ }^{34-38}$. Testing of CSF can miss pathogens present in CNS tissue which may require a brain biopsy to identify. Neuroimaging should be utilized for stereotactic brain biopsy localization with consideration given to targeting affected areas with the least chance of affecting functional outcomes. Brain biopsy specimens should undergo histopathologic evaluation, staining for pathogens, cultures, and flash-freezing for targeted PCR testing or metagenomic sequencing. 


\section{Therapeutic Approach}

Supportive care is the mainstay of encephalitis therapy with careful management of the airway in cases of severely altered mental status or loss of bulbar function, management of intracranial pressure and cerebral edema, fluid and electrolyte management, and seizure control with anti-epileptic medications. While targeted therapies should be tailored towards the specific etiology once identified, a structured approach to administering empiric therapies for common treatable causes is warranted while diagnostic evaluation is in process. In patients in whom it is difficult to differentiate bacterial meningitis from viral meningoencephalitis, intravenous antibiotics (eg. vancomycin and a third-generation cephalosporin at meningeal dosing) should be promptly initiated. Antibiotics should be initiated after lumbar puncture and CSF cultures are obtained, if deemed safe and performed immediately. All children with suspected encephalitis should be started on empiric intravenous acyclovir $(20 \mathrm{mg} / \mathrm{kg} /$ dose every 8 hours for age $<3$ months and $10 \mathrm{mg} / \mathrm{kg} / \mathrm{dose}$ every 8 hours for age $>3$ months in the presence of normal renal function) while undergoing diagnostic evaluation for $\mathrm{HSV}^{1,39}$. Acyclovir should be continued until HSV testing is negative, and consideration should be given to continuing acyclovir while pursuing repeat CSF testing and/or brain biopsy in cases with high clinical suspicion with no alternative etiology identified ${ }^{1,21}$.

Few viral etiologies of encephalitis have therapies proven to be effective for immunocompetent children. If HSV is detected (or highly suspected), intravenous acyclovir should be administered for 3 weeks with repeat lumbar puncture and HSV PCR testing near the end of therapy to ensure clearance before stopping therapy ${ }^{1,39}$. In neonates, this should be followed by 6 months of oral acyclovir suppressive therapy to reduce recurrences and improve neurodevelopmental outcomes ${ }^{40}$. In patients aged $>12$ years, a three month course of oral valacyclovir did not provide added neuropsychological benefit following standard intravenous treatment with acyclovir ${ }^{41}$. Acyclovir is frequently administered for VZVassociated encephalitis; however, efficacy is unproven and it remains unclear if this is a direct viral infection or an immune-mediated post-infectious process. Oral influenza antivirals, such as oseltamivir, are recommended for hospitalized children with influenza identified in respiratory specimens, including those with encephalitis, though effectiveness for CNS disease is unknown. No effective therapies are currently available for the treatment of encephalitis due to EVs, most non-influenza respiratory viruses, arboviruses, or rabies virus in immunocompetent children; experimental treatments are potential options for some agents (eg. adenovirus, rabies virus).

Identified bacterial, fungal, and parasitic CNS infections warrant treatment with targeted antimicrobial therapy, based on known antimicrobial susceptibilities when available. Treatment of non-CNS bacterial infections associated with encephalitis or encephalopathy, such as Mycoplasma pneumoniae and Bordetella pertussis respiratory infections, can be considered, though impact on the course of CNS disease has not been studied.

The use of corticosteroids, intravenous immune globulin (IVIG), and plasma exchange (PLEX) has not been systematically studied with controlled trials in encephalitis ${ }^{42}$. Due to differing pathophysiology amongst the various etiologies (ie. active CNS infection vs. post- 
infectious or non-infectious immune-mediated), care should be taken when considering immunosuppressive therapies such as corticosteroids, and potentially PLEX, until the potential for active infections has been sufficiently assessed or is being treated with effective antimicrobial therapy. During the process of diagnostic evaluation for potential infectious encephalitis, IVIG carries the least potential immunosuppressive risk of these modalities. Additionally, IVIG may assist with pathogen clearance in children with humoral immunodeficiency, as well as immune modulation with certain pathogens, such as EV$\mathrm{A} 71^{43}$. When a diagnosis of ADEM is probable, first line therapy is high-dose intravenous corticosteroids, with PLEX and IVIG considerations for refractory disease $e^{42,44,45}$. Immunotherapy, including corticosteroids, IVIG and PLEX alone or combined, and tumor removal (if present) are the mainstays of treatment for anti-NMDAR encephalitis, with second line options including rituximab and cyclophosphamide ${ }^{46}$.

\section{Prevention}

Vaccination against poliovirus, measles virus, mumps virus, seasonal influenza virus, and pertussis is recommended as part of the immunization series for children and likely provide protection against encephalitis associated with these pathogens. Travelers should be evaluated for eligibility to receive immunization against vaccine-preventable endemic diseases associated with encephalitis in the region to which they are traveling (ie. Japanese encephalitis virus, rabies virus, enterovirus A71, and tickborne encephalitis virus). Protection against mosquito and tick bites, including staying in screened facilities, wearing of long sleeves, use of repellants with proven efficacy, public health measures such as standing water pool mitigation and pesticide application in outbreak situations, is recommended to decrease the risk of arboviral encephalitis ${ }^{47}$. Use of sterile water for sinus irrigation and avoiding recreational activities in warm freshwater are the only certain methods of prevention for Naeglaeria fowlerii encephalitis ${ }^{48}$. Post-exposure prophylaxis is recommended with rabies vaccine series and rabies immunoglobulin for rabies-prone animal bites, valacyclovir for old world Macaque monkey bites to decrease risk of Herpes B encephalitis, albendazole for exposure to raccoon feces or latrines to decrease risk of baylisascariasis, and acyclovir for neonates born to mothers with active genital herpes lesions at the time of delivery ${ }^{49}$.

\section{Disease Course, Prognosis, and Outcomes}

Nearly all children with encephalitis in the US are hospitalized with $40 \%$ requiring critical care in an intensive care unit. Prolonged length of stay, averaging 16 days up to 25 days in those requiring intensive care, and need for inpatient rehabilitation services $(\sim 20-40 \%)$ are common $^{10,50}$. The average cost of acute hospitalization of a child with encephalitis in the US is estimated to be between $\$ 64,000$ to $\$ 260,000$ depending on the level of care and rehabilitation needs required ${ }^{10,51}$.

Most children with encephalitis have incomplete recovery at discharge and those who fully recover are most likely to do so within $6-12$ months ${ }^{50,52}$. Though there is limited data on long-term neurologic outcomes in children, persistent long-term neurologic sequelae, including learning problems, developmental delays, and behavioral problems are 
common $^{50-53}$. The subsequent development of epilepsy is more common in those who presented initially with seizures and is correlated with long-term neurologic sequelae $e^{50,53,54}$. Children with abnormal neuroimaging are less likely to fully recover and report poorer quality of life at long-term followup ${ }^{50,51}$.

Outcomes differ greatly based on etiology. Many children with HSV encephalitis have longterm neurologic impairment, particularly those with delayed initiation of acyclovir ${ }^{52,55-57}$. Neonates with EV encephalitis have variable outcomes ranging from full recovery to significant long-term deficits, while older infants and children with EV encephalitis tend to demonstrate significant recovery (with the exception of EV-A71) ${ }^{52}$. Encephalitis due to $\mathrm{HPeVs}$ in young, particularly pre-term, infants may have more long-term neurodevelopmental sequelae than encephalitis due to $\mathrm{EVs}^{58,59}$. Nearly $80 \%$ of children with anti-NMDAR encephalitis will have full or substantial response to immunotherapy, though $25 \%$ will have subsequent relapse ${ }^{60}$.

Recent population-based studies estimate a 3\% mortality rate of encephalitis in children in the $\mathrm{US}^{9,10}$. Complications requiring intensive care including respiratory failure, intubation, sepsis, and pneumonia are predictors of mortality ${ }^{9}$. HSV is the most common cause of pediatric death due to encephalitis, though some rare causes, such as amebic and rabies encephalitis are nearly uniformly fatal ${ }^{9,26}$. Mortality is rare from most EVs (except EVA71), HPeVs, arboviruses (except eastern equine encephalitis virus), and autoantibodymediated encephalitides in children $8,25,60,61$.

\section{References}

1. Tunkel AR, Glaser CA, Bloch KC, et al. The management of encephalitis: clinical practice guidelines by the Infectious Diseases Society of America. Clin Infect Dis. 2008; 47(3):303-327. [PubMed: 18582201]

2. Flett KB, Rao S, Dominguez SR, Bernard T, Glode MP. Variability in the Diagnosis of Encephalitis by Pediatric Subspecialists: The Need For a Uniform Definition. J Pediatric Infect Dis Soc. 2013; 2(3):267-269. [PubMed: 26619481]

3. Venkatesan A, Tunkel AR, Bloch KC, et al. Case definitions, diagnostic algorithms, and priorities in encephalitis: consensus statement of the international encephalitis consortium. Clin Infect Dis. 2013; 57(8):1114-1128. [PubMed: 23861361]

4. Sejvar JJ, Kohl KS, Bilynsky R, et al. Encephalitis, myelitis, and acute disseminated encephalomyelitis (ADEM): case definitions and guidelines for collection, analysis, and presentation of immunization safety data. Vaccine. 2007; 25(31):5771-5792. [PubMed: 17570566]

5. Kestenbaum LA, Ebberson J, Zorc JJ, Hodinka RL, Shah SS. Defining cerebrospinal fluid white blood cell count reference values in neonates and young infants. Pediatrics. 2010; 125(2):257-264. [PubMed: 20064869]

6. Seiden JA, Zorc JJ, Hodinka RL, Shah SS. Lack of cerebrospinal fluid pleocytosis in young infants with enterovirus infections of the central nervous system. Pediatr Emerg Care. 2010; 26(2):77-81. [PubMed: 20093996]

7. Abzug MJ, Levin MJ, Rotbart HA. Profile of enterovirus disease in the first two weeks of life. The Pediatric infectious disease journal. 1993; 12(10):820-824. [PubMed: 8284118]

8. Renaud C, Harrison CJ. Human Parechovirus 3: The Most Common Viral Cause of Meningoencephalitis in Young Infants. Infect Dis Clin North Am. 2015; 29(3):415-428. [PubMed: 26188604]

9. George BP, Schneider EB, Venkatesan A. Encephalitis hospitalization rates and inpatient mortality in the United States, 2000-2010. PloS one. 2014; 9(9):e104169. [PubMed: 25192177] 
10. Bagdure D, Custer JW, Rao S, et al. Hospitalized Children With Encephalitis in the United States: A Pediatric Health Information System Database Study. Pediatric neurology. 2016; 61:58-62. [PubMed: 27353693]

11. Iro MA, Sadarangani M, Goldacre R, Nickless A, Pollard AJ, Goldacre MJ. 30- year trends in admission rates for encephalitis in children in England and effect of improved diagnostics and measles-mumps-rubella vaccination: a population-based observational study. The Lancet Infectious diseases. 2017

12. Wickstrom R, Fowler A, Bogdanovic G, Bennet R, Eriksson M. Review of the aetiology, diagnostics and outcomes of childhood encephalitis from 1970 to 2009. Acta Paediatr. 2017; 106(3):463-469. [PubMed: 27886400]

13. Pahud BA, Glaser CA, Dekker CL, Arvin AM, Schmid DS. Varicella zoster disease of the central nervous system: epidemiological, clinical, and laboratory features 10 years after the introduction of the varicella vaccine. The Journal of infectious diseases. 2011; 203(3):316-323. [PubMed: 21177308]

14. Lewis P, Glaser CA. Encephalitis. Pediatrics in review / American Academy of Pediatrics. 2005; 26(10):353-363.

15. Britton PN, Dale RC, Booy R, Jones CA. Acute encephalitis in children: Progress and priorities from an Australasian perspective. J Paediatr Child Health. 2015; 51(2):147-158. [PubMed: 24953748]

16. Pruss H, Finke C, Holtje M, et al. N-methyl-D-aspartate receptor antibodies in herpes simplex encephalitis. Annals of neurology. 2012; 72(6):902-911. [PubMed: 23280840]

17. Armangue T, Moris G, Cantarin-Extremera V, et al. Autoimmune post-herpes simplex encephalitis of adults and teenagers. Neurology. 2015; 85(20):1736- 1743. [PubMed: 26491084]

18. Glaser CA, Gilliam S, Schnurr D, et al. In search of encephalitis etiologies: diagnostic challenges in the California Encephalitis Project, 1998-2000. Clin Infect Dis. 2003; 36(6):731-742. [PubMed: 12627357]

19. Gable MS, Sheriff H, Dalmau J, Tilley DH, Glaser CA. The frequency of autoimmune N-methylD-aspartate receptor encephalitis surpasses that of individual viral etiologies in young individuals enrolled in the California Encephalitis Project. Clin Infect Dis. 2012; 54(7):899-904. [PubMed: 22281844]

20. Glaser CA, Honarmand S, Anderson LJ, et al. Beyond viruses: clinical profiles and etiologies associated with encephalitis. Clin Infect Dis. 2006; 43(12):1565- 1577. [PubMed: 17109290]

21. Weil AA, Glaser CA, Amad Z, Forghani B. Patients with suspected herpes simplex encephalitis: rethinking an initial negative polymerase chain reaction result. Clin Infect Dis. 2002; 34(8):11541157. [PubMed: 11915008]

22. de Crom SC, Obihara CC, de Moor RA, Veldkamp EJ, van Furth AM, Rossen JW. Prospective comparison of the detection rates of human enterovirus and parechovirus RT-qPCR and viral culture in different pediatric specimens. J Clin Virol. 2013; 58(2):449-454. [PubMed: 23973350]

23. Perez-Velez CM, Anderson MS, Robinson CC, et al. Outbreak of neurologic enterovirus type 71 disease: a diagnostic challenge. Clin Infect Dis. 2007; 45(8):950-957. [PubMed: 17879907]

24. Leber AL, Everhart K, Balada-Llasat JM, et al. Multicenter Evaluation of the BioFire FilmArray Meningitis Encephalitis Panel for the Detection of Bacteria, Viruses and Yeast in Cerebrospinal Fluid Specimens. Journal of clinical microbiology. 2016

25. Gaensbauer JT, Lindsey NP, Messacar K, Staples JE, Fischer M. Neuroinvasive arboviral disease in the United States: 2003 to 2012. Pediatrics. 2014; 134(3):e642-650. [PubMed: 25113294]

26. Capewell LG, Harris AM, Yoder JS, et al. Diagnosis, Clinical Course, and Treatment of Primary Amoebic Meningoencephalitis in the United States, 1937-2013. J Pediatric Infect Dis Soc. 2015; 4(4):e68-75. [PubMed: 26582886]

27. Schuster FL, Yagi S, Gavali S, et al. Under the radar: balamuthia amebic encephalitis. Clin Infect Dis. 2009; 48(7):879-887. [PubMed: 19236272]

28. Cope JR, Ali IK. Primary Amebic Meningoencephalitis: What Have We Learned in the Last 5 Years? Curr Infect Dis Rep. 2016; 18(10):31. [PubMed: 27614893] 
29. Chow FC, Glaser CA, Sheriff H, et al. Use of clinical and neuroimaging characteristics to distinguish temporal lobe herpes simplex encephalitis from its mimics. Clin Infect Dis. 2015; 60(9):1377-1383. [PubMed: 25637586]

30. Beattie GC, Glaser CA, Sheriff H, et al. Encephalitis with thalamic and basal ganglia abnormalities: etiologies, neuroimaging, and potential role of respiratory viruses. Clin Infect Dis. 2013; 56(6):825-832. [PubMed: 23196954]

31. Kincaid O, Lipton HL. Viral myelitis: an update. Current neurology and neuroscience reports. 2006; 6(6):469-474. [PubMed: 17074281]

32. Messacar K, Schreiner TL, Van Haren K, et al. Acute flaccid myelitis: A clinical review of US cases 2012-2015. Annals of neurology. 2016; 80(3):326-338. [PubMed: 27422805]

33. Krupp LB, Banwell B, Tenembaum S. International Pediatric MSSG. Consensus definitions proposed for pediatric multiple sclerosis and related disorders. Neurology. 2007; 68(16 Suppl 2):S7-12. [PubMed: 17438241]

34. Wilson MR, Naccache SN, Samayoa E, et al. Actionable diagnosis of neuroleptospirosis by nextgeneration sequencing. The New England journal of medicine. 2014; 370(25):2408-2417. [PubMed: 24896819]

35. Greninger AL, Messacar K, Dunnebacke T, et al. Clinical metagenomic identification of Balamuthia mandrillaris encephalitis and assembly of the draft genome: the continuing case for reference genome sequencing. Genome Med. 2015; 7:113. [PubMed: 26620704]

36. Naccache SN, Peggs KS, Mattes FM, et al. Diagnosis of neuroinvasive astrovirus infection in an immunocompromised adult with encephalitis by unbiased next-generation sequencing. Clin Infect Dis. 2015; 60(6):919-923. [PubMed: 25572898]

37. Phan TG, Messacar K, Dominguez SR, da Costa AC, Deng X, Delwart E. A new densovirus in cerebrospinal fluid from a case of anti-NMDA-receptor encephalitis. Arch Virol. 2016; 161(11): 3231-3235. [PubMed: 27522586]

38. Mongkolrattanothai K, Naccache SN, Bender JM, et al. Neurobrucellosis: Unexpected Answer From Metagenomic Next-Generation Sequencing. J Pediatric Infect Dis Soc. 2017

39. Kimberlin DW, Lin CY, Jacobs RF, et al. Safety and efficacy of high-dose intravenous acyclovir in the management of neonatal herpes simplex virus infections. Pediatrics. 2001; 108(2):230-238. [PubMed: 11483782]

40. Kimberlin DW, Whitley RJ, Wan W, et al. Oral acyclovir suppression and neurodevelopment after neonatal herpes. The New England journal of medicine. 2011; 365(14):1284-1292. [PubMed: 21991950]

41. Gnann JW Jr, Skoldenberg B, Hart J, et al. Herpes Simplex Encephalitis: Lack of Clinical Benefit of Long-term Valacyclovir Therapy. Clin Infect Dis. 2015; 61(5):683-691. [PubMed: 25956891]

42. Esposito S, Picciolli I, Semino M, Principi N. Steroids and childhood encephalitis. The Pediatric infectious disease journal. 2012; 31(7):759-760. [PubMed: 22695191]

43. Wang SM, Lei HY, Huang MC, et al. Modulation of cytokine production by intravenous immunoglobulin in patients with enterovirus 71-associated brainstem encephalitis. J Clin Virol. 2006; 37(1):47-52. [PubMed: 16861032]

44. Schwartz J, Winters JL, Padmanabhan A, et al. Guidelines on the use of therapeutic apheresis in clinical practice-evidence-based approach from the Writing Committee of the American Society for Apheresis: the sixth special issue. J Clin Apher. 2013; 28(3):145-284. [PubMed: 23868759]

45. Graus F, Titulaer MJ, Balu R, et al. A clinical approach to diagnosis of autoimmune encephalitis. The Lancet Neurology. 2016; 15(4):391-404. [PubMed: 26906964]

46. Titulaer MJ, McCracken L, Gabilondo I, et al. Treatment and prognostic factors for long-term outcome in patients with anti-NMDA receptor encephalitis: an observational cohort study. The Lancet Neurology. 2013; 12(2):157-165. [PubMed: 23290630]

47. Centers for Disease Control and Prevention. [Accessed July 19, 2017, 2017] Mosquito Prevention. 2017. https://www.cdc.gov/features/stopmosquitoes/

48. Centers for Disease Control and Prevention. [Accessed July 19, 2017, 2017] Prevention of Naegleria fowlerii. 2017. https://www.cdc.gov/parasites/naegleria/prevention.html

49. Committee on Infectious Diseases. Red Book. 30. American Academy of Pediatrics; 2015. 
50. Rao S, Elkon B, Flett KB, et al. Long-Term Outcomes and Risk Factors Associated With Acute Encephalitis in Children. J Pediatric Infect Dis Soc. 2015

51. DuBray K, Anglemyer A, LaBeaud AD, et al. Epidemiology, outcomes and predictors of recovery in childhood encephalitis: a hospital-based study. The Pediatric infectious disease journal. 2013; 32(8):839-844. [PubMed: 23518823]

52. Fowler A, Stodberg T, Eriksson M, Wickstrom R. Long-term outcomes of acute encephalitis in childhood. Pediatrics. 2010; 126(4):e828-835. [PubMed: 20876179]

53. Rismanchi N, Gold JJ, Sattar S, et al. Neurological Outcomes After Presumed Childhood Encephalitis. Pediatric neurology. 2015; 53(3):200-206. [PubMed: 26220354]

54. Rismanchi N, Gold JJ, Sattar S, et al. Epilepsy After Resolution of Presumed Childhood Encephalitis. Pediatric neurology. 2015; 53(1):65-72. [PubMed: 26092415]

55. Rautonen J, Koskiniemi M, Vaheri A. Prognostic factors in childhood acute encephalitis. The Pediatric infectious disease journal. 1991; 10(6):441-446. [PubMed: 1906597]

56. Ward KN, Ohrling A, Bryant NJ, Bowley JS, Ross EM, Verity CM. Herpes simplex serious neurological disease in young children: incidence and long-term outcome. Archives of disease in childhood. 2012; 97(2):162-165. [PubMed: 21685219]

57. Lahat E, Barr J, Barkai G, Paret G, Brand N, Barzilai A. Long term neurological outcome of herpes encephalitis. Archives of disease in childhood. 1999; 80(1):69- 71. [PubMed: 10325763]

58. Britton PN, Dale RC, Nissen MD, et al. Parechovirus Encephalitis and Neurodevelopmental Outcomes. Pediatrics. 2016

59. Vergnano S, Kadambari S, Whalley K, et al. Characteristics and outcomes of human parechovirus infection in infants (2008-2012). Eur J Pediatr. 2015; 174(7):919-924. [PubMed: 25573462]

60. Florance NR, Davis RL, Lam C, et al. Anti-N-methyl-D-aspartate receptor (NMDAR) encephalitis in children and adolescents. Annals of neurology. 2009; 66(1):11-18. [PubMed: 19670433]

61. Fowlkes AL, Honarmand S, Glaser C, et al. Enterovirus-associated encephalitis in the California encephalitis project, 1998-2005. The Journal of infectious diseases. 2008; 198(11):1685-1691. [PubMed: 18959496]

62. Kimberlin DW, Baley J. Committee on Infectious D, Committee on F Newborn. Guidance on management of asymptomatic neonates born to women with active genital herpes lesions. Pediatrics. 2013; 131(2):383-386. [PubMed: 23378604]

63. To TM, Soldatos A, Sheriff H, et al. Insights into pediatric herpes simplex encephalitis from a cohort of 21 children from the California Encephalitis Project, 1998-2011. The Pediatric infectious disease journal. 2014; 33(12):1287-1288. [PubMed: 24911898]

64. Abzug MJ. The enteroviruses: problems in need of treatments. The Journal of infection. 2014; 68(Suppl 1):S108-114. [PubMed: 24119825]

65. Sells CJ, Carpenter RL, Ray CG. Sequelae of central-nervous-system enterovirus infections. The New England journal of medicine. 1975; 293(1):1-4. [PubMed: 1168853]

66. Messacar K, Breazeale G, Wei Q, Robinson CC, Dominguez SR. Epidemiology and clinical characteristics of infants with human parechovirus or human herpes virus-6 detected in cerebrospinal fluid tested for enterovirus or herpes simplex virus. Journal of medical virology. 2015

67. Karsch K, Obermeier P, Seeber L, et al. Human Parechovirus Infections Associated with Seizures and Rash in Infants and Toddlers. The Pediatric infectious disease journal. 2015; 34(10):1049_ 1055. [PubMed: 26181895]

68. Verboon-Maciolek MA, Groenendaal F, Hahn CD, et al. Human parechovirus causes encephalitis with white matter injury in neonates. Annals of neurology. 2008; 64(3):266-273. [PubMed: 18825694]

69. Armangue T, Petit-Pedrol M, Dalmau J. Autoimmune encephalitis in children. Journal of child neurology. 2012; 27(11):1460-1469. [PubMed: 22935553]

70. Pohl D, Alper G, Van Haren K, et al. Acute disseminated encephalomyelitis: Updates on an inflammatory CNS syndrome. Neurology. 2016; 87(9 Suppl 2):S38-45. [PubMed: 27572859]

71. Murthy SN, Faden HS, Cohen ME, Bakshi R. Acute disseminated encephalomyelitis in children. Pediatrics. 2002; 110(2 Pt 1):e21. [PubMed: 12165620] 
72. Davis LE, Booss J. Acute disseminated encephalomyelitis in children: a changing picture. The Pediatric infectious disease journal. 2003; 22(9):829-831. [PubMed: 14506377]

73. Sejvar JJ. Acute disseminated encephalomyelitis. Curr Infect Dis Rep. 2008; 10(4):307-314. [PubMed: 18765104]

74. Granerod J, Ambrose HE, Davies NW, et al. Causes of encephalitis and differences in their clinical presentations in England: a multicentre, population-based prospective study. The Lancet Infectious diseases. 2010; 10(12):835-844. [PubMed: 20952256] 


\section{KEY POINTS}

- 1 : Encephalitis is an uncommon and potentially devastating condition of neurologic dysfunction due to brain parenchymal inflammation

- $\quad 2$ : In the absence of brain biopsy, presence of encephalopathy with clinical findings suggestive of central nervous system inflammation infers a diagnosis of encephalitis.

- $\quad 3$ : Viruses, including herpes simplex viruses and enteroviruses, are the most common causes in children in the US, although immune-mediated etiologies are increasingly recognized and may respond to immune modulation.

- 4: Given the broad differential diagnosis, a staged diagnostic approach can be initially targeted towards common, treatable, and at-risk etiologies, followed by broader, more invasive testing for unexplained persistent or severe disease.

- 5: Supportive care with empiric therapy towards bacteria and herpes simplex viruses should be administered during diagnostic evaluation with definitive therapy ultimately tailored towards identified treatable etiologies. 


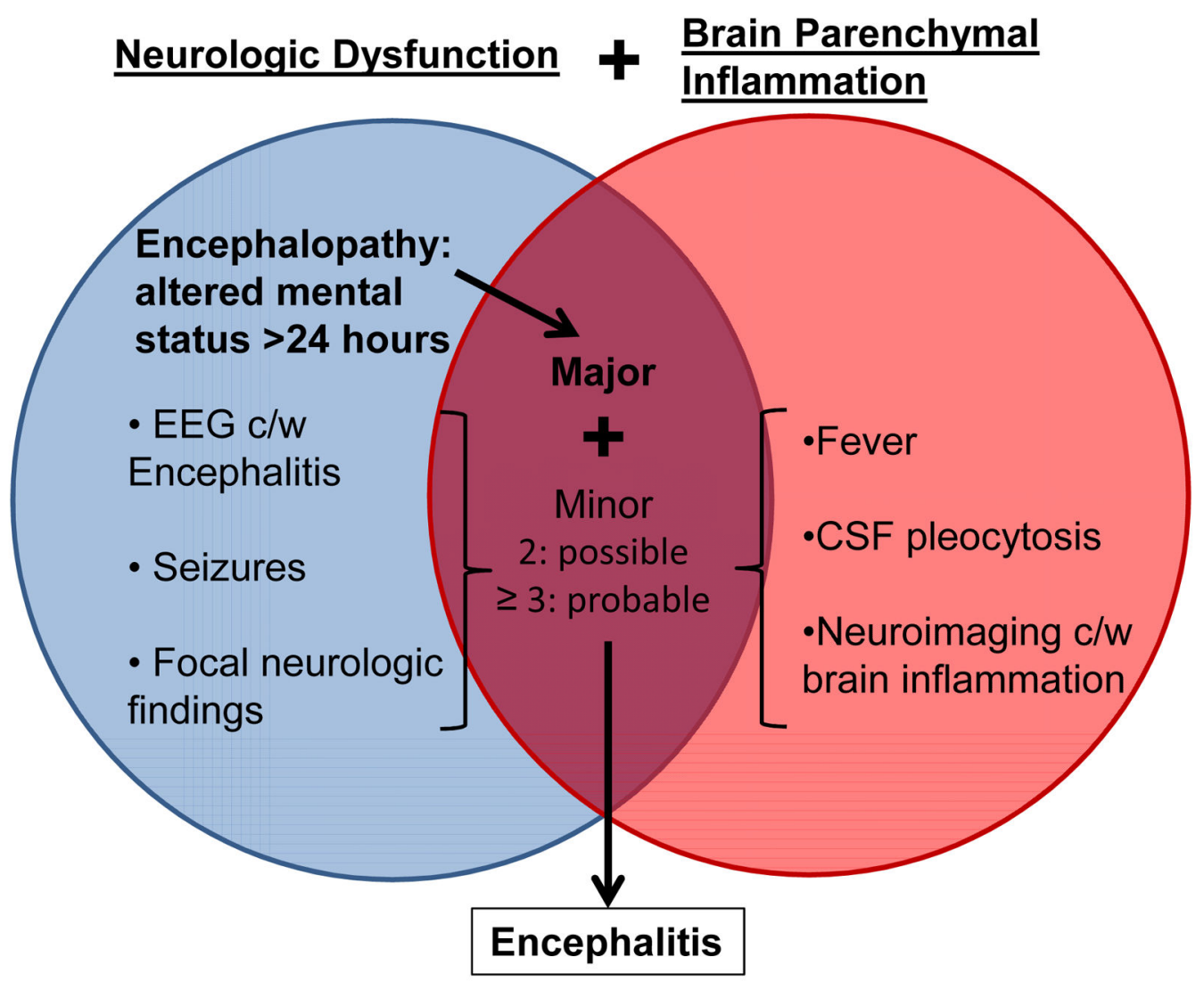

Figure 1. Overview of Diagnostic Criteria for Encephalitis

The major criterion required to meet the case definition for encephalitis is altered mental status $>24$ hours without alternative cause identified. Two additional minor criteria are required for possible encephalitis, wherease three or more additional minor criteria are required for probable encephalitis. Confirmed encephalitis additionally requires brain tissue pathology with inflammation, a diagnosis of a microorganism strongly associated with encephalitis, or laboratory evidence of an autoimmune condition strongly associated with encephalitis. Abbreviations: $\mathrm{EEG}=$ electroencephalogram, $\mathrm{c} / \mathrm{w}=$ consistent with, $\mathrm{CSF}=$ cerebrospinal fluid 


\section{Table 1}

\section{Summary of Common Viral Causes of Encephalitis in Children}

Herpes Simplex Virus (HSV): HSV is the most common infectious etiology of encephalitis in children, driven largely by cases of perinatally acquired HSV-2 encephalitis ${ }^{9}$. Neonatal HSV encephalitis is most common in the first month of life with fever, temperature instability, lethargy, seizures, vesicles, or sepsis-like illness. Pregnant women with active genital HSV lesions at delivery should receive acyclovir and deliver via Csection, when possible, to decrease risk of perinatal transmission ${ }^{49}$. Infants born to mothers with active genital HSV lesions at delivery should receive acyclovir while undergoing HSV evaluation ${ }^{49,62}$. HSV evaluation in neonates includes CSF HSV PCR, as well as surface swab and blood HSV testing and liver function testing (LFTs) ${ }^{49}$. Older children commonly present with lethargy, fever, confusion and seizures. Most children with HSV encephalitis eventually have abnormal CSF with mild lymphocytic pleocytosis and elevated protein, though early samples can be normal ${ }^{63}$. MRI may demonstrate temporal and extratemporal lobe lesions and/or hemorrhage ${ }^{29}$. Diagnosis of HSV encephalitis is made by PCR of CSF (or brain tissue) which may be negative early in the course ${ }^{63}$. Treatment is three weeks of intravenous acyclovir, which in neonates should be followed by 6 months of oral acyclovir ${ }^{40}$. Though early antiviral treatment improves outcomes, HSV encephalitis carries a significant risk of mortality and many surviving neonates and children will have neurodevelopmental sequelae.

Enterovirus (EV): EV is the second most common cause of encephalitis in children, most frequently occurring in the first year of life, and largely driven by the predominant circulating serotype(s) for the given year ${ }^{9,61}$. Neonatal EV encephalitis typically follows maternal EV illness preceding or immediately following delivery as part of a systemic neonatal EV sepsis syndrome. Children with humoral immunodeficiencies (eg. hypogammaglobulinemia) are at higher risk of EV encephalitis and may have chronic, difficult to clear infections. Children with EV meningoencephalitis can present with fever, headaches, meningism, exanthem and/or enanthem with seizures, focal neurologic changes or alterations in consciousness ${ }^{64}$. EV encephalitis is diagnosed by PCR of CSF, though with certain EVs (eg. poliovirus, EV-A71, EV-D68) CSF may be negative and detection may require testing of throat, rectal, blood, or respiratory specimens ${ }^{23}$. IVIG has not been studied specifically in children with encephalitis, though has shown efficacy in hypogammaglobulinemic children with chronic EV encephalitis. No effective antivirals are currently available ${ }^{64}$. Supportive care remains the mainstay of treatment. Unlike meningitis due to EVs, encephalitis carries increased risk of neurologic compromise ${ }^{65}$.

Human Parechoviruses (HPeV): HPeVs are recently recognized as important and common causes of viral encephalitis in young infants. Infants may present with high fevers, sepsis-like illness, seizures, or lethargy. Peripheral leukopenia, elevated LFTs, and a palm-sole rash can be suggestive of $\mathrm{HPeV}$ infection ${ }^{66,67}$. CSF typically lacks pleocytosis. Periventricular white matter lesions on MRI are characteristic of HPeV encephalitis ${ }^{68}$. HPeV encephalitis is diagnosed by HPeV-specific CSF PCR and can also be detected from blood, throat and rectal swabs. Supportive care is the mainstay of therapy as there are no known effective treatments. Though most infants will show short-term improvement, long-term neurodevelopmental sequelae have been described ${ }^{58}$.

Arboviruses $^{25}$ : Arboviruses, including La Crosse virus (LACV), West Nile virus (WNV), Eastern equine encephalitis virus (EEEV), Powassan virus (POWV), and St Louis encephalitis virus (SLEV) remain important causes of seasonal encephalitis in children. LACV is the most common cause of arboviral encephalitis in US children, primarily affecting younger children (mean age 7 years) in Appalachian and Midwestern regions. WNV encephalitis is much less common in children than adults and has widespread distribution throughout the US. EEEV is a rare cause of severe encephalitis in children (33\% case-fatality rate), mostly along the Atlantic and Gulf coasts. POWV is an increasingly recognized tick-borne arboviral cause of encephalitis, most common in the upper midwestern US in the spring months. SLEV caused intermittent epidemics of encephalitis every 10-20 years in the US, but may be replaced by WNV due to cross-reactive immunity in birds, the zoonotic host. Arbovirus diagnosis is made by virus-specific IgM testing of serum (probable case) and CSF (confirmed case). Clinical management is supportive as there are no specific treatments. 
Table 2

\section{Summary of Selected Immune-Mediated Causes of Encephalitis in Children}

Acute Disseminated Encephalomyelitis (ADEM): ADEM is an inflammatory demyelinating CNS condition; it is the most commonly identified immune mediated encephalitis with highest incidence in early childhood (mean 5-8 years) ${ }^{69,70}$. A temporal association with a prodromal illness (commonly upper respiratory tract infection), or rarely vaccination, in the preceding 3 weeks can often be identified ${ }^{71,72}$. Clinical symptoms include acute onset encephalopathy with multifocal sensory and motor deficits which depend on the location of the lesions in the brain and spinal cord ${ }^{71}$. MRI of the brain and spine demonstrate asymmetric, bilateral, poorly marginated, hyperintense lesions in the subcortical white and deep gray matter ${ }^{70}$. Over half have abnormal CSF, most commonly mild lymphocytic pleocytosis with elevated protein ${ }^{72}$. Oligoclonal bands are rarely found, unlike in multiple sclerosis 70,73 . Around $25 \%$ of children will have myelin oligodendrocyte gylcoprotein antibodies, which are associated with relapse and optic neuritis ${ }^{70}$. First line therapy is high dose intravenous corticosteroids with second line IVIG or PLEX ${ }^{70}$. Mortality is rare with around $80 \%$ of children fully recovering with treatment ${ }^{71}$.

Anti-N-methyl-D-aspartate Receptor (anti-NMDAR) Encephalitis: Anti-NMDAR encephalitis is the second leading cause of identified immune-mediated encephalitis in children ${ }^{74}$. In teenagers, a prodromal illness is followed days to weeks later by psychiatric and behavioral symptoms progressing to encephalopathy, seizures, and abnormal movements. Young children eventually develop a similar syndrome but may have more prominent initial behavioral changes (particularly agitation and aggression), speech changes, personality changes, sleep changes, seizures, and movement disorders (particularly gait disturbance) ${ }^{60,69}$. Dysautonomia and hypoventilation are less common in children than in adults $^{60}$. MRI is abnormal in less than half of cases, but CSF is abnormal in most (lymphocytic pleocytosis and possibly elevated protein or oligoclonal bands) and EEG is abnormal in nearly all (diffuse background slowing, focal slowing, or seizures) ${ }^{69}$. Diagnosis is confirmed by antibodies to the NR1 subunit of NMDAR found in serum or CSF, the latter being more specific, with level of titer correlating with prognosis. Though tumor is rare in children ${ }^{60}$ (particularly males), if identified, removal improves outcome ${ }^{69}$. Immunotherapy includes first line treatment with corticosteroids, IVIG, PLEX, or a combination, and secondary options include rituximab and cyclophosphamide ${ }^{46,69}$. Around $80 \%$ of patients have substantial recovery ${ }^{46}$, though relapse is seen in around $25 \%$ of children ${ }^{60}$. 
Table 3

Diagnostic and Therapeutic Approach to Encephalitis in US Children

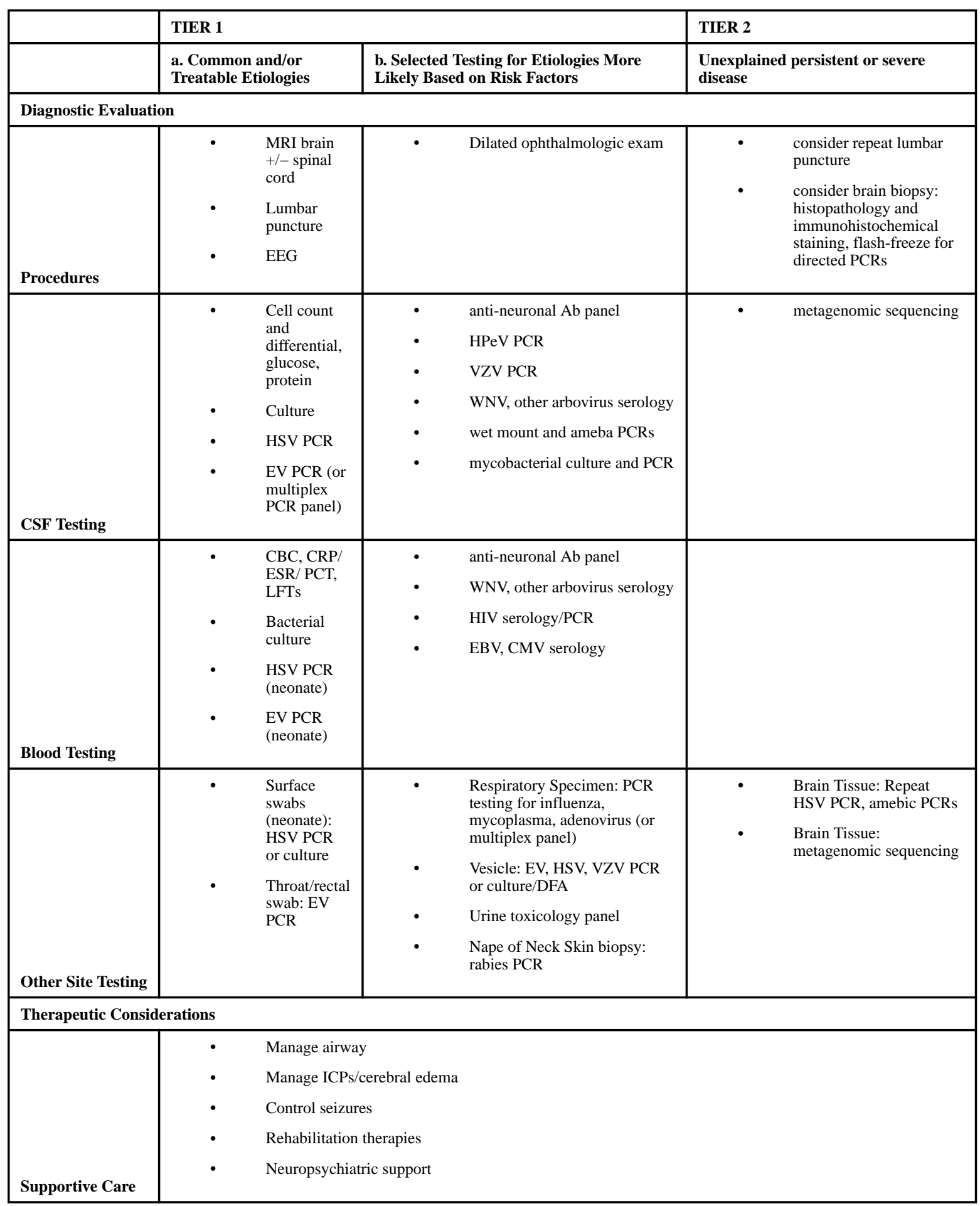

Infect Dis Clin North Am. Author manuscript; available in PMC 2019 March 01. 


\begin{tabular}{|c|c|c|c|c|c|c|}
\hline \multirow[b]{3}{*}{ 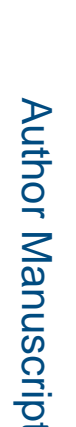 } & & \multicolumn{4}{|l|}{ TIER 1} & \multirow{2}{*}{$\begin{array}{l}\text { TIER } 2 \\
\begin{array}{l}\text { Unexplained persistent or severe } \\
\text { disease }\end{array}\end{array}$} \\
\hline & & \multicolumn{2}{|c|}{$\begin{array}{l}\text { a. Common and/or } \\
\text { Treatable Etiologies }\end{array}$} & \multicolumn{2}{|c|}{$\begin{array}{l}\text { b. Selected Testing for Etiologies More } \\
\text { Likely Based on Risk Factors }\end{array}$} & \\
\hline & Medications & • & $\begin{array}{l}\text { Acyclovir } \\
\text { until HSV } \\
\text { ruled out } \\
\text { Antibiotics } \\
\text { until } \\
\text { bacterial } \\
\text { meningitis } \\
\text { ruled out }\end{array}$ & • & $\begin{array}{l}\text { Targeted therapy based on } \\
\text { identified etiology } \\
\text { Corticosteroids/IVIG/PLEX if } \\
\text { immune-mediated encephalitis } \\
\text { suspected and potential for } \\
\text { active infection sufficiently } \\
\text { assessed }\end{array}$ & \\
\hline
\end{tabular}

Abbreviations: $\mathrm{PCR}=$ polymerase chain reaction, $\mathrm{DFA}=$ Direct fluorescent antibody, $\mathrm{ICP}=$ intracranial pressure, $\mathrm{HSV}=\mathrm{herpes}$ simplex virus, $\mathrm{EV}=$ enterovirus, $\mathrm{HPeV}=$ human parechovirus, $\mathrm{VZV}=$ varicella zoster virus, $\mathrm{WNV}=$ West Nile virus, $\mathrm{EBV}=$ Epstein-Barr virus, CMV=cytomegalovirus, HIV=human immunodeficiency virus 
Table 4

Directed Differential Diagnosis of Encephalitis in US Children

\begin{tabular}{|c|c|c|}
\hline Risk Category & Established Etiology & Rare cause/mimic \\
\hline \multicolumn{3}{|l|}{ Age } \\
\hline Neonates/infants & HSV, CMV, EV, HPeV & $\begin{array}{l}\text { Toxoplasma gondii, Treponema } \\
\text { pallidum, Zika virus }\end{array}$ \\
\hline Teenagers & HIV, EBV, WNV & Treponema pallidum \\
\hline \multicolumn{3}{|l|}{ Vaccination History } \\
\hline Unvaccinated & Measles, Mumps, Rubella, VZV, Poliovirus & \\
\hline Recently Vaccinated & ADEM & \\
\hline \multicolumn{3}{|l|}{ Season } \\
\hline Summer/fall & EV, WNV, LACV & $\begin{array}{l}\text { Rickettsia, Ehrlichia, Anaplasma, } \\
\text { Borrelia burgdorferi }\end{array}$ \\
\hline Winter & Influenza, adenovirus & respiratory viruses \\
\hline \multicolumn{3}{|l|}{ International Travel } \\
\hline Endemic regions & Rabies, JEV, TBEV, Measles & $\begin{array}{l}\text { TB, dengue, chikungunya, Zika, } \\
\text { Plasmodium }\end{array}$ \\
\hline \multicolumn{3}{|l|}{ Animal Exposure } \\
\hline Bat/Skunk bite & Rabies & \\
\hline Raccoon feces & Baylisascaris procyonis & \\
\hline Rodent & LCMV & \\
\hline Cat & Bartonella henselae & \\
\hline Mosquito & WNV, LACV, EEEV, SLEV & \\
\hline Tick & Powassan & $\begin{array}{l}\text { Rickettsia, Ehrlichia, Anaplasma, } \\
\text { Borrelia burgdorferi }\end{array}$ \\
\hline \multicolumn{3}{|l|}{ Activity } \\
\hline Freshwater swimming & Naegleria fowleri, Acanthamoeba & \\
\hline Eating Unpasteurized Dairy & Listeria monocytogenes & Toxoplasma, Brucella, Coxiella \\
\hline Eating Undercooked Meat & & Toxoplasma \\
\hline $\begin{array}{l}\text { Eating Undercooked Seafood, } \\
\text { Unwashed Vegetables }\end{array}$ & Angiostrongylus sp. & \\
\hline \multicolumn{3}{|l|}{ Clinical Findings } \\
\hline Vesicular Rash & HSV, VZV, EV & \\
\hline Lymphadenopathy & $\begin{array}{l}\text { Generalized: EBV, CMV, HIV, Localized: } \\
\text { Bartonella }\end{array}$ & \\
\hline Psychiatric/behavioral Changes & Rabies, anti-NMDAR & \\
\hline Respiratory symptoms & Mycoplasma, influenza, adenovirus & respiratory viruses \\
\hline Retinitis or keratitis & WNV, CMV, Bartonella & $\begin{array}{l}\text { Toxoplasma gondii, Treponema } \\
\text { pallidum, Toxocara canis/catis }\end{array}$ \\
\hline Parotitis & Mumps, influenza, respiratory viruses & \\
\hline Eosinophilic CSF & Angiostrongylus sp. & $\begin{array}{l}\text { Baylisascaris procyonis, Naegleria } \\
\text { fowleri, Balamuthia mandrillaris, } \\
\text { Acanthamoeba, TB, fungi, Toxocara } \\
\text { canis/catis }\end{array}$ \\
\hline
\end{tabular}




\begin{tabular}{|l|l|l|}
\hline Risk Category & Established Etiology & Rare cause/mimic \\
\hline Imaging/EEG Localization & & \\
\hline Frontal lobe & Naegleria fowleri & \\
\hline Temporal lobe & HSV & \\
\hline Thalamus/basal ganglia & WNV, influenza & Respiratory viruses \\
\hline Cerebellum & VZV & \\
\hline Brainstem/basilar & Listeria monocytogenes, EV-A71, HSV & TB \\
\hline Myelitis & Poliovirus, non-polio EVs, WNV, JEV & \\
\hline
\end{tabular}

Bolded organisms signify potentially treatable pathogens. Rare/cause mimics include pathogens which are rare causes of encephalitis in immunocompetent US children or which cause a clinical syndrome which mimics encephalitis. Abbreviations: HSV=herpes simplex virus, $\mathrm{CMV}=$ cytomegalovirus, $\mathrm{EV}=$ enterovirus, $\mathrm{HPeV}=$ human parechovirus, $\mathrm{HIV}=$ human immunodeficiency virus, EBV=Epstein-Barr virus, WNV=West Nile virus, LACV=La Crosse virus VZV=varicella zoster virus, ADEM=acute disseminated encephalomyelitis, JEV=Japanese encephalitis virus, $\mathrm{TBEV}=$ tick-borne encephalitis virus, $\mathrm{TB}=$ tuberculosis, $\mathrm{LCMV}=$ lymphocytic choriomeningitis virus, $\mathrm{EEEV=eastern} \mathrm{equine}$ encephalitis virus, SLEV=St. Louis encephalitis virus, anti- NMDAR=anti-N-methyl-D-aspartate receptor encephalitis. 